by systems and deals with them all concisely, competently and in an orderly fashion. A surprising amount of information is thus contained in short chapters, which are enhanced by the really excellent line drawings by Miss Sweet. This is bound to be a popular book and deservedly so.

A. G. SIGNY

SARCOMAS OF THE BRAIN By James W. Kernohan and Alfred Uihlein (viii +192 ; 96 figures. \$8.0) Springfield, Illinois: Charles C. Thomas. 1962.

The authors describe, largely from the files of the Mayo Clinic, 241 sarcomas of the brain out of a total of 8,070 intracerebral tumours. Even from such a vast number of brain growths most British pathologists would be surprised at the number of sarcomas identified and the major feeling which the volume will arouse will centre around the justification for including some of the described growths under the heading of sarcoma. Doubt about the nomenclature detracts little from the value of the book which gives an excellent account of some unusual tumours of the brain which are difficult to classify anyway, but which occur with sufficient frequency to justify a distinctive nomenclature.

The authors give a brief historical account of each of the six types of brain sarcoma they have accepted and are careful to present the views of other authorities who have reached different conclusions about the origins of some of the growths. The numerous different names under which the growths have been described are given along with a considerable bibliography so that apart from anything else the book is a valuable reference for alternative names to some unusual intracerebral growths.

They classify brain sarcomata into fibrosarcomas, giant cell sarcomas, circumscribed sarcomas of the cerebellum, meningeal sarcomatosis, haemangiopericytomas, and sarcomas of the reticuloendothelial system, the latter divided into reticuloendothelial-cell sarcomas, Hodgson's sarcomas, and microgliomas. Of the major groups, many pathologists will be reluctant to accept the giant cell sarcomas as an entity separate from glioblastoma multiforme, and the haemangiopericytoma from the angioblastic meningioma. There will be little dispute about the fibrosarcoma arising from the meninges or within the brain, for some growths in these situations cannot be distinguished from fibrosarcomas which would be readily accepted in any other part of the body. Whether, however, astrocytic gliomas with apparent sarcomatous elements should be regarded as mixed gliosarcomas is a matter of some doubt. Few would challenge their cases of sarcomas of the reticuloendothelial system occuring within the brain, nor diffuse meningeal sarcomatosis for cases of uniform invasion of the meninges by sarcomatous growths occur in which the most scrupulous and careful search reveals no primary in the brain or elsewhere. In children one is inclined to think that a small medulloblastoma may have been missed, but the appearance of some diffuse meningeal growths in children is somewhat different from diffusely spread medulloblastomas. Without doubt also the growth described as a circumscribed sarcoma of the cerebellum presents a histological appearance which merits its separation from other cerebellar growths although instances of this neoplasm must often have been designated as medulloblastomas. Its relatively more frequent occurrence in adult life, coupled with its distinctive histological features, establishes the case for placing this growth in a separate category.

In all their accounts the authors have given valuable clinicopathological correlations and studied the prognosis of the cases they have been able to follow. The book is, therefore, as complete an account of the tumours that might be called sarcomas of the brain as one could obtain. British pathologists, influenced by the tendency to simple nomenclature of tumours, may react with some initial antagonism to the additional diagnoses suggested in this book, but to anyone interested in the accurate identification of brain growths this volume provides much valuable information and is clearly the result of great experience and thought. The majority of the illustrations are good and demonstrate well the points made in the text.

B. E. TOMLINSON

ANAEROBIC BACTERIOLOGY IN CLINICAL MEDICINE, 2nd ED.

By A. Trevor Willis. (xiv $+234 ; 18$ tables. $47 s 6 d$ )

London: Butterworths. 1964.

The first edition of this book has proved deservedly popular as a laboratory handbook. In the preface the author says that he was stimulated to write the book by 'the dread often expressed by students and colleagues about the difficulties of growing anaerobes.' With the clear exposition of laboratory methods for the growth and identification of anaerobes presented in the first half of this book, no bacteriologist should find the subject too difficult for him. The second half of the book gives a clear and concise account of the essentials of anaerobic infections and the toxicology of anaerobes, together with the guiding principles of prophylactic and curative treatment.

The new edition is essentially similar, with certain additions. The most notable of these are a considerable increase in space allotted to the anaerobic cocci, largely on the basis of the work of Professor Hare and his colleagues; a much fuller bibliography; and a discussion. In relation to the latter the author is firmly in favour of using tetanus antitoxin for prophylaxis 'for all persons with soiled wounds, including women who have had a criminal abortion', if they have not been previously immunized. He of course goes on to point out the necessity for taking precautions against hypersensitivity phenomena, and adds that if antitoxin be given, either for prophylactic or curative purposes, 'subsequent active immunization must be mandatory, and should be the responsibility of the physician who orders the antitoxin to be given.'

The new edition also contains descriptions of a few extra culture media, a fuller account of clinical cases of infection, and recent work on the lecithinase and lipase activity of Clostridia. The brief sections on the Fusiform group remain the least satisfactory parts of the book, but as the author points out Cowan and Steel 'found it convenient to omit them from their diagnostic 
tables' published in 1961 and in their recent book (1965) the latter authors admit that the classification of this genus 'is not well established'.

\section{MARY BARBER}

COMMON COLDS AND RELATED DISEASE By D. A. J. Tyrrell. (pp. 197; 49 figures. 42s.) London: 1965 Edward Arnold.

This is a new book and a companion volume for Professor Stuart-Harris's book on influenza. Dr. Tyrrell has set out to piece together a mass of new data on the common cold and related respiratory infections and in doing so has achieved a nice balance of material that will be found useful to both clinician and laboratory worker. In the first six chapters, Dr. Tyrrell discusses the clinical syndrome, epidemiology, and volunteer studies on the common cold and methods for studying respiratory viruses in the laboratory. Subsequent chapters are devoted to the myxoviruses, adenoviruses, reoviruses and the common cold or rhinoviruses. A useful chapter is included on prophylaxis and treatment. The book contains numerous charts and illustrations, all of which are extremely well produced. This is a welcome and useful addition to textbooks outlining modern trends in virology and is thoroughly recommended to both clinicians and virologists.

\section{J. A. DUDGEON}

INFLUENZA AND OTHER VIRUS INFECTIONS OF THE RESPIRATORY TRACT, 2nd ED. By C. H. Stuart-Harris, with a foreword by Sir Christopher Andrewes (Pp. vii + 248; 25 tables. 45s.) London: Edward Arnold.

The first edition of Professor Stuart-Harris's monograph, published in 1953, described four main clinical syndromes associated with acute respiratory infections-influenza, the common cold, atypical pneumonia, and febrile catarrh. At that time, apart from influenza, our knowledge of the aetiology of these conditions depended on human volunteer experiments. Now, eleven years later, this monograph has had to be entirely rewritten, such has been the advances in our knowledge of respiratory viruses. Many new viruses have been discovered, the adenoviruses, parainfluenza viruses, and respiratory syncytial, all of which are fully discussed in this new edition together with methods of laboratory diagnosis by tissue culture and serology. The account of the clinical symptoms produced by these numerous causative agents are excellent. It is well documented and with good illustrations. Two chapters on treatment and prevention put the problem of chemotherapy and immunization into clear perspective. An amazing amount of fresh information has been fitted into this new edition and it is thoroughly recommended for all those who wish to keep abreast of these new developments.

\section{J. A. DUDGEON}

DYNAMIC STUDIES OF METABOLIC BONE DISEASE. Edited by O. H. Pearson and G. F. Joplin. (viii + 229; illustrated 50s.) Oxford:1964 Blackwell Scientific Publications. This slim but attractive volume contains the proceedings of a symposium which was held at Western Reserve
University, U.S.A., in March 1963 on four related topics. The first section deals with the interpretation of calcium isotope studies, the second with osteoporosis, the third $\%$ with osteomalacia and Paget's disease, and the fourth $\overrightarrow{\overline{\vec{S}}}$ with the excretion of hydroxyproline.

For the average clinical pathologist the approach will읃 be somewhat esoteric since the papers deal with some $\overline{\bar{O}}$ of the most controversial aspects of what is in itself a $\frac{\bar{\omega}}{\bar{D}}$ highly controversial field. On the other hand, this book must be considered to be essential reading for anyone engaged in this field or proposing to enter it. Bauer's $\$$ introductory review on tracer techniques is useful and $\overrightarrow{0}$ straightforward. Heaney's contribution on the interpre-tation of calcium kinetic data is also admirable and so is $\vec{\omega}$ his description of his studies of disuse osteoporosis. Lafferty and Pearson describe again their controversialo procedures for the measurement of bone resorption and $\vec{\Delta}$ present some remarkable results in vitamin-D resistant rickets. Arnold's work on the quantitation of bone $\sigma$ mineralization in osteoporosis is admirable and is followed by Urist's description of accelerated aging and $\dot{\circ}$ premature death of bone cells in osteoporosis. Finally Klein and Curtiss review the use of urinary hydroxyproline as an index of bone metabolism but seem reluc- $\vec{c}$ tant to accept the general view that this reflects collagen $\mathbb{D}$ breakdown rather than collagen synthesis.

This is a useful contribution to a difficult and rapidly $\frac{\mathbb{D}}{3}$ changing field. The individual papers still remain fresh although written two years ago. The book is profusely illustrated and perhaps this and its somewhat limitê $\overrightarrow{0}$ appeal may explain its relatively high cost.

B. E. C. NORDIN

ADVANCES IN METABOLIC DISORDERS, vol 1, edited by

R. Levine and R. Luft. (Pp. xxii +366 ; illustrated. 86s.) New York and London: Academic Press. 1964.

This is the first volume of a new series primarily of impor- $\stackrel{\mathbb{Q}}{\circ}$ tance to chemical pathologists but not unimportant to pathologists in other branches and to physicians. Eight $\frac{0}{3}$ subjects are treated: glycogen storage disease, the para- $\frac{\supset}{J}$ thyroids, mitochondrial respiratory control, osteoporosis, basal metabolic rate and thyroid hormones, insulin antagonists and inhibitors, aldosterone, and folic acid $\overline{ }$ deficiency and its interrelationship with vitamin $\mathbf{B}_{12}$. metabolism. The chapters on the parathyroids and on 3 osteoporosis both cover well-trodden ground and the chapter on mitochondrial respiratory control contains $\frac{0}{3}$ much that is little more than hypothesis to explain well- $₹$ established facts. The remaining chapters can fairly be을 described as excellent reviews of their particular topics.

Books and journals have multiplied so extensively음 that one is reluctant to say that no one can afford to be without a given book, but it may apply to this one. ARTHUR JORDAN

TECHNiQues in Chemical PATHology By G. A. Cheyne. (Pp. vii + 397; 14 plates; 24 figures. 42s.) Oxford:웅 Blackwell Scientific Publications. 1964.

This book, stated to be primarily aimed at technicians taking the examinations of the Institute of Medical Laboratory Technology, consists of two parts. The first, $\square$ occupying almost a third of the book, is devoted to 